\title{
Glomerular Capillary
}

National Cancer Institute

\section{Source}

National Cancer Institute. Glomerular Capillary. NCI Thesaurus. Code C32684.

Tiny blood vessels located within Bowman's capsule, which combine in networks to form a glomerulus. 